\title{
Impact of Intravenous Aspirin Administration on Ventriculostomy-Associated Hemorrhage in Coiled Acute Subarachnoid Hemorrhage Patients
}

\author{
David Evans, BSc, MBBCh, PGCert ${ }^{1}$, Richard Flood, BMBS, FRCR ${ }^{1}$, Owain Davies, BSc, MBChB, MRCS ${ }^{2}$, \\ James Wareham, BSc, MBChB, FRCR ${ }^{1}$, Alex Mortimer, PhD, MRCS, FRCR ${ }^{1}$ \\ ${ }^{1}$ Department of Neuroradiology, North Bristol NHS Trust, Bristol, UK \\ ${ }^{2}$ Department of Neurosurgery, North Bristol NHS Trust, Bristol, UK
}

\begin{abstract}
Purpose: Aspirin has beneficial effects on coiling, even in acute subarachnoid hemorrhage, but there is also a perceived risk of increased bleeding and, importantly, a concern regarding ventriculostomy-associated hemorrhage (VAH) in those with complicating hydrocephalus. We aimed to assess the rate and extent of VAH in patients specifically treated with procedural intravenous aspirin during endovascular coiling of ruptured intracranial aneurysms.
\end{abstract}

Materials and Methods: This was a single neurovascular center retrospective observational study of consecutive patients treated over a three-year period. The rate of VAH assessed using computed tomography and clinical outcomes were compared in patients receiving intraprocedural intravenous aspirin loading $(n=90)$ versus those that did not receive the drug $(n=40)$.

Results: There was a significantly elevated rate of VAH in patients receiving intravenous aspirin (30\% vs. 2.5\%, odds ratio 16.7 [95\% confidence interval: 2.2-128.0], $P<0.0001$ ). The majority of VAH was $<10 \mathrm{~mm}$ in size (70\%) with the largest bleed measuring $20 \mathrm{~mm}$. No hematoma required surgical evacuation. No difference in favorable outcome at discharge was demonstrated. There was no difference in mortality between the 2 groups.

Conclusion: Loading with intravenous aspirin during endovascular treatment of ruptured intracranial aneurysms significantly increases the risk of VAH, but most are small with minimal impact on clinical outcome at discharge. Intravenous aspirin should probably be reserved for selected cases but should not be withheld based on risk of VAH.

Key Words: Aneurysm; Aspirin; External ventricular drain; Ventriculostomy; Antiplatelet; Subarachnoid haemorrhage

\section{INTRODUCTION}

Thromboembolic events during or after endovascular coiling of ruptured cerebral aneurysms are reduced by aspirin use, ${ }^{1,2}$ and treatment of wide-necked aneurysms, coil protrusion, or use of adjunctive devices such as stents or flow-diverters commonly necessitates anti-platelet use to avoid such complications. Furthermore, post-procedural aspirin has also been shown to improve clinical outcomes in a randomized trial of clipped and coiled subarachnoid hemorrhage patients, though the mechanism for this remains uncertain. ${ }^{3}$

\section{Correspondence to:}

Alex Mortimer, PhD, MRCS, FRCR

Department of Neuroradiology,

Southmead Hospital, North Bristol

NHS Trust, Southmead Road, Bristol,

BS10 5NB, UK

Tel: +44 (0) 1174149015

E-mail: alex.mortimer@nbt.nhs.uk

Received: March 14, 2021

Revised: June 11, 2021

Accepted: June 15, 2021
Copyright $\odot 2021$ Korean Society of Interventional Neuroradiology

This is an Open Access article distributed under the terms of the Creative Commons Attribution Non-Commercial License (http://creativecommons.org/licenses/by-nc/4.0) which permits unrestricted non-commercial use, distribution, and reproduction in any medium, provided the original work is properly cited. 
Evidence for an impact on delayed ischaemic process is conflicting, ${ }^{3-5}$ but there remains a school of thought that aspirin may limit the deleterious effects of at least part of this process. Nevertheless, routine use of procedural intravenous (IV) aspirin loading to compliment treatment of ruptured intracranial aneurysms remains controversial, largely owing to a presumed increase in bleeding risk.

Platelet function is depressed in a proportion of patients with acute subarachnoid hemorrhage, and aspirin compounds this through its action on the potent platelet aggregant thromboxane A2 via inhibition of cyclooxygenase-1. ${ }^{6}$ Intravenous aspirin also demonstrates a rapid onset of action (minutes vs. hours) when compared to the orally administered drug. ${ }^{7}$ It is plausible that early aneurysm rebleeding could be more common in those treated with intra-procedural aspirin, ${ }^{8}$ and a further concern in this patient group is for ventriculostomy-associated hemorrhage (VAH). ${ }^{9}$ Reported rates of $\mathrm{VAH}$ range from $10 \%$ to $43 \%,{ }^{10-13}$ and antiplatelet use has been identified as a risk factor for $V A H .{ }^{9}$ The aim of this study was to assess the impact of intra-procedural IV aspirin loading during acute endovascular treatment of ruptured intracranial aneurysms on the rate and extent of VAH.

\section{MATERIALS AND METHODS}

This study was approved by the Institutional Review Board (IRAS 256424). This was a single-center retrospective observational analysis of patients presenting with aneurysmal subarachnoid hemorrhage treated using endovascular techniques between 2015 and 2018. Patients were included in the primary study population if ventricular drainage was required for hydrocephalus and further dichotomized into groups based on whether they received procedural IV aspirin loading or not. The primary outcome measure was the rate of VAH. The secondary outcome measures were of in-hospital mortality and favorable clinical outcome at discharge dichotomized as Glasgow outcome scale (GOS) 4 or 5.

Ventriculostomy was performed by the acute neurosurgical team, most commonly as an emergency procedure at presentation. A ventricular catheter was passed into the lateral ventricle using anatomical landmarks or image guidance, with a right or left frontal approach dependant on ventricular size and intraventricular blood load. The ventricular catheters were then tunneled subcutaneously away from the insertion site and connected to an external drainage system with variable high to control cerebrospinal fluid drainage. If ventricular catheters required revision for blockage or infection, the same technique was used.

All aneurysms were treated by consultant interventional neuroradiologists within 48 hours of presentation. Procedures were performed using systemic heparinisation. The decision to use procedural aspirin was based on operator preference; some operators preferred routine procedural IV aspirin use while other operators reserved IV aspirin for widenecked lesions, coil prolapse, or adjunctive stent use. Aspirin was administered intravenously at a dose of 300-1,000 mg (most commonly 500 mg) after initial coil placement.
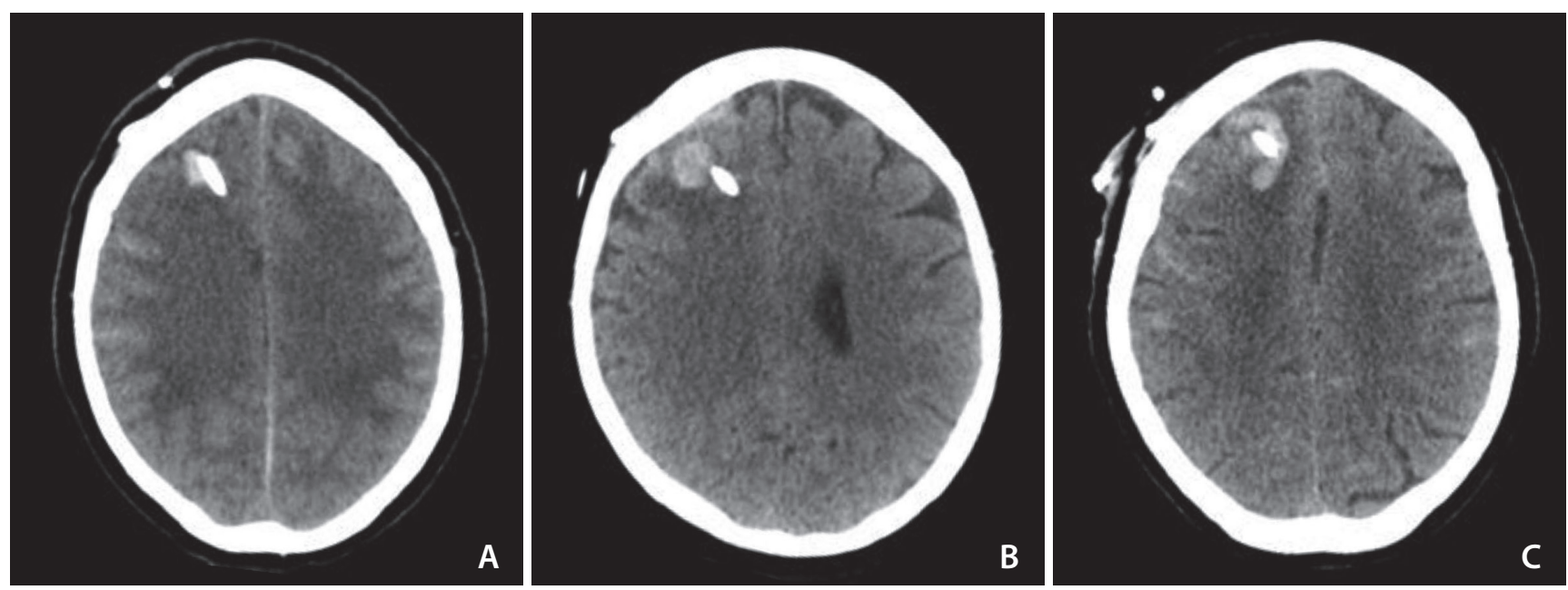

Fig. 1. Characteristic ventriculostomy-associated hemorrhage (VAH). (A) A typical $<1 \mathrm{~cm}$ hematoma that accounted for the vast majority of $V A H$. (B) A $13 \mathrm{~mm}$ hematoma typical of the larger bleeds seen in the study. (C) The largest hematoma encountered in this study measuring $20 \mathrm{~mm}$ in the longest dimension. 
Post-procedural aspirin use was again based on operator preference and commenced the following day at a dose of $75 \mathrm{mg}$ orally or via a nasogastric tube. In summary, some patients were loaded with IV aspirin during the procedure and then commenced on post-procedural low-dose aspirin, whereas others were treated with either low-dose post-procedural aspirin alone (commenced more than 12 hours after coiling) or no aspirin at all.

A prospectively maintained departmental database was used to obtain patient demographics, presenting World Federation of Neurological Surgeons (WFNS) grade, Glasgow Coma Scale (GCS), Glasgow Outcome Scale (GOS), and inpatient mortality. Electronic patient records, the hospital radiology information system, and the hospital picture archiving and communication system were used to obtain data such as procedure dates and prior use of anticoagulants or antiplatelets.

Admission and subsequent imaging including non-contrast computed tomography (CT), CT angiography, and digital subtraction angiography performed during the inpatient episode were assessed for modified Fisher grade, degree of hydrocephalus (EVANS index), distribution of intraventricular haemorrhage (IVH), extent of lateral ventricular hemorrhage (graded 1-4; 1: <25\% IVH filling of lateral ventricles; 2: IVH filling $25-50 \%$ of lateral ventricles; 3 : IVH filling $50-75 \%$ of lateral ventricles; 4: IVH filling $>75 \%$ of lateral ventricles), aneurysm location and size, adjunctive stent use, and hemorrhagic complications. Ventriculostomy-associated hemorrhage was defined as any new parenchymal or intra-ventricular high density on subsequent $C T$ scans adjacent to the drain of at least $5 \mathrm{~mm}$ diameter in any plane (Fig $1 \mathrm{~A}-\mathrm{C}$ ).

Data were statistically analyzed using Graphpad software (San Diego, CA, USA). Non-parametric data were assessed using Fisher's exact test and ordinal data with the KruskalWallis test. Parametric data were assessed using the t-test. In our analysis, a P-value of $<0.05$ was regarded as statistically significant.

\section{RESULTS}

In total, 355 patients were treated during the study period. A

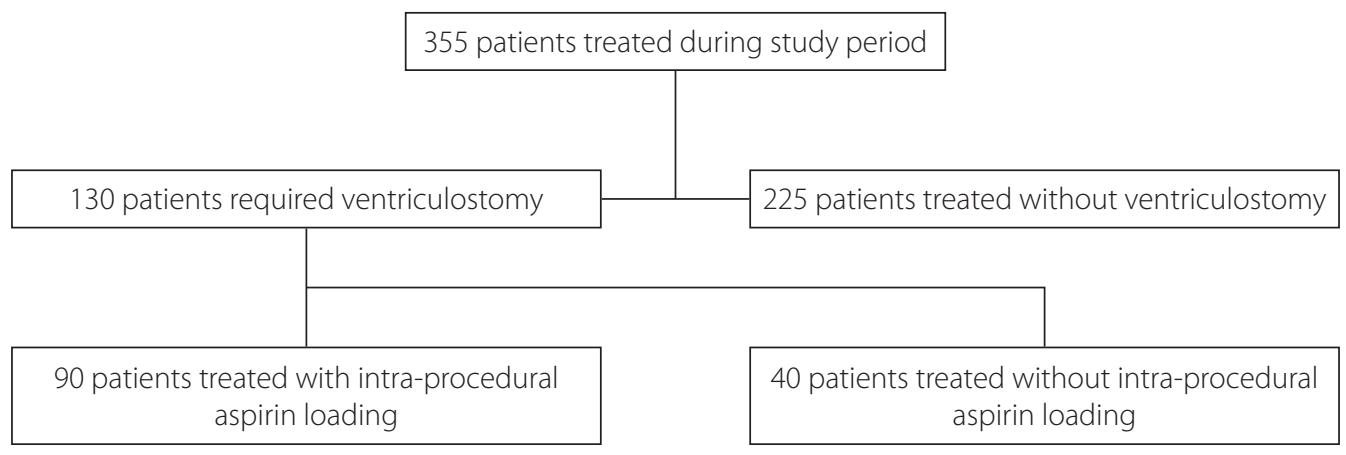

Fig. 2. Summary of patient selection.

Table 1. Baseline clinical characteristics

\begin{tabular}{lccc}
\hline Variable & Procedural IV aspirin loading $(n=90)$ & No procedural aspirin $(n=40)$ & P-value \\
\hline Age $(y r)$ & $62.2 \pm 11.5$ & $61.1 \pm 11.7$ & 0.618 \\
Sex, female & $72(80.0)$ & $31(77.5)$ & 0.816 \\
WFNS 1 & $16(17.8)$ & $6(15.0)$ & 0.803 \\
WFNS 2 & $16(17.8)$ & $7(17.5)$ & 1.000 \\
WFNS 3 & $13(14.4)$ & $6(15.0)$ & 1.000 \\
WFNS 4 & $30(33.3)$ & $11(27.5)$ & 0.547 \\
WFNS 5 & $15(16.7)$ & $10(25.0)$ & 0.335 \\
GCS, median (IQR) & $12(5.5)$ & $10.5(7.3)$ & 0.384 \\
\hline
\end{tabular}

Values are presented as mean \pm standard deviation or number (\%).

IV, intravenous; WFNS, World Federation of Neurological Surgeons; GCS, Glasgow coma scale; IQR, interquartile range. 
summary of patient selection is shown in Fig. 2. Of them, 225 (63.3\%) were treated without needing external ventricular drain insertion; the patients in this group were predominantly of good grade (WFNS 1-2 in 91.6\%). The primary study population (those who had undergone ventriculostomy insertion) comprised 130 patients. Ninety patients received procedural IV aspirin loading and 40 did not. Ninety-eight percent of those receiving IV aspirin received further lowdose aspirin commencing more than 12 hours after the procedure had ended. Sixty percent of the 40 patients who were not loaded with IV aspirin were subsequently administered low-dose aspirin.

There was no significant difference in baseline demographics or severity of presenting clinical grade (Table 1); of those treated with IV aspirin, 64.4\% were WFNS grade 3-5, whereas $67.5 \%$ were WFNS grade 3-5 in the group who did not receive IV aspirin loading. There was no significant difference between the 2 groups in terms of extent of hydrocephalus or baseline subarachnoid or intraventricular hemorrhage (Table 2). There tended to be larger aneurysms in the IV aspi-

Table 2. Baseline neuroradiological characteristics

\begin{tabular}{|c|c|c|c|}
\hline Variable & Procedural IV aspirin loading $(n=90)$ & No procedural aspirin $(n=40)$ & P-value \\
\hline \multicolumn{4}{|l|}{ Aneurysm size (mm) } \\
\hline$<7$ & $53(58.9)$ & $29(72.5)$ & 0.170 \\
\hline $7-12$ & $31(34.4)$ & $7(17.5)$ & 0.061 \\
\hline $13-24$ & $6(6.7)$ & $2(5.0)$ & 1.000 \\
\hline Ant circulation & $77(85.6)$ & $33(82.5)$ & 0.793 \\
\hline Wide neck & $31(34.4)$ & $15(37.5)$ & 0.843 \\
\hline Stent-assisted & $14(15.6)$ & $0(0)$ & 0.005 \\
\hline Modified Fisher, median (IQR) & $4(0)$ & $4(0)$ & 0.481 \\
\hline Evans index, median (IQR) & $0.3(0.04)$ & $0.3(0.05)$ & 0.874 \\
\hline Lateral ventricular IVH grade, median (IQR) & $2(1)$ & $2(1)$ & 0.851 \\
\hline 3rd ventricular haemorrhage & $57(63.3)$ & $24(60.0)$ & 0.850 \\
\hline 4th ventricular haemorrhage & $60(66.7)$ & $28(70.0)$ & 0.840 \\
\hline
\end{tabular}

Values are presented as number (\%).

IV, intravenous; IQR, interquartile range; IVH, intraventricular haemorrhage.

Table 3. Periprocedural characteristics

\begin{tabular}{|c|c|c|c|}
\hline Variable & $\begin{array}{l}\text { Procedural IV aspirin loading } \\
\qquad(\mathrm{n}=90)\end{array}$ & $\begin{array}{l}\text { No procedural aspirin } \\
\qquad(n=40)\end{array}$ & P-value \\
\hline Antiplatelet use at presentation & $7(7.8)$ & $6(15.0)$ & 0.220 \\
\hline Anticoagulation at presentation & $0(0)$ & $1(2.5)$ & 1.000 \\
\hline Drain placement prior to endovascular procedure & $73(81.1)$ & $33(82.5)$ & 1.000 \\
\hline Drain placed within $24 \mathrm{~h}$ of coiling procedure & $67(74.4)$ & $32(80.0)$ & 0.516 \\
\hline \multicolumn{4}{|l|}{ IV aspirin dose (mg) } \\
\hline $250-300$ & $5(5.5)$ & - & - \\
\hline 500 & $83(92.2)$ & - & - \\
\hline 1,000 & $2(2.2)$ & - & - \\
\hline $\begin{array}{l}\text { Low dose }(75 \mathrm{mg} \text { ) Aspirin commenced }>12 \mathrm{~h} \\
\text { post-procedure }\end{array}$ & $88(97.8)$ & $24(60.0)$ & $<0.001$ \\
\hline Post-procedural dual anti-platelet therapy & $3(3.3)$ & $1(2.5)$ & 1.000 \\
\hline
\end{tabular}

Values are presented as number (\%).

$\mathrm{IV}$, intravenous. 
rin group, but this was not statistically significant (Table 2). Of the patients who were given IV aspirin, 14 procedures were stent-assisted. No stent-assisted procedures were performed in the group that did not receive IV aspirin ( $\mathrm{P}=0.005)$.

Periprocedural features are displayed in Table 3. More than $90 \%$ of patients in the IV aspirin group were administered a dose of $500 \mathrm{mg}$. Ventriculostomy was performed prior to the endovascular procedure in $81 \%$ and $82.5 \%$ of cases in each treatment group, respectively.

Clinical and radiological outcomes are summarised in Table 4. Those administered IV aspirin had a higher rate of VAH (30\% vs. 2.5\%, odds ratio 16.7 [95\% confidence interval: 2.2-128.0], $\mathrm{P}<0.0001)$. There was also a greater rate of $\mathrm{VAH}>10 \mathrm{~mm}$ diameter in the IV aspirin group (8.9\% vs. 0\%) but this was not statistically significant $(P=0.106)$. No hematoma required surgical evacuation. No hematoma resulted in significant mass effect. There was no significant difference in the rate of patients requiring multiple ventricular drains or in the rate of ventriculitis. No difference in the rate of subsequent shunt insertion or length of hospital stay was demonstrated. One patient in the IV aspirin group was recognized to have a periprocedural thromboembolic event, whereas no patients in the group without IV aspirin administration suffered a periprocedural thrombotic event.

No difference in favorable outcome at discharge was demonstrated (47.8\% were GOS 4-5 in those administered IV aspirin versus 50\% in those who did not receive the drug). There was no difference in mortality between the 2 groups.

\section{DISCUSSION}

Immediate benefits of aspirin use during endovascular coiling include treatment of or prophylactic reduction in thromboembolic events and facilitation of the use of stents to treat aneurysms of more challenging morphology. ${ }^{1,2}$ However, it is well established that antiplatelet agents will increase the risk of ventriculostomy associated bleeding. ${ }^{9,14,15}$ A combination of aspirin and clopidogrel has been shown to have a more significant impact in prior studies but single-agent therapy with aspirin has been deemed low risk for symptomatic bleeding. ${ }^{9}$

The aim of this observational study was to determine specifically whether, in the context of acute subarachnoid hemorrhage, administration of intravenous aspirin during endovascular treatment of ruptured intracranial aneurysms within 48 hours of the ventriculostomy procedure significantly increases the risk of ventriculostomy-associated hemorrhage and whether this bleeding alters clinical outcome (only a tiny minority of patients in this study received an additional agent, whereas many other studies included patients with dual anti-platelets in this context). Aspirin is likely to reach a therapeutic level far quicker when administered intravenous$l_{1}^{7}$ and we hypothesized that this speed of action coupled with a relatively short time between ventriculostomy (when there is conceivably some physical blood-brain barrier disruption) and IV aspirin administration (<24 hours in 74.4\%) may be critical to development of VAH in these patients.

While within the range of hemorrhage rates published in the medical literature, ${ }^{10-13}$ the results of this study suggest

Table 4. Clinical and radiological outcomes for patients treated with a bolus of intraprocedural aspirin versus those without an intraprocedural bolus

\begin{tabular}{|c|c|c|c|}
\hline Variable & $\begin{array}{l}\text { Procedural IV aspirin loading } \\
\qquad(\mathrm{n}=90)\end{array}$ & $\begin{array}{l}\text { No procedural aspirin use } \\
\qquad(n=40)\end{array}$ & P-value \\
\hline Ventriculostomy associated haemorrhage & $27(30.0)$ & $1(2.5)$ & $<0.001$ \\
\hline Ventriculostomy associated haemorrhage $\geq 10 \mathrm{~mm}$ & $8(8.9)$ & $0(0)$ & 0.106 \\
\hline Ventriculostomy associated haemorrhage $\geq 20 \mathrm{~mm}$ & $1(1.1)$ & $0(0)$ & 1.000 \\
\hline GOS $4-5$ at discharge & $43(47.8)$ & $20(50.0)$ & 0.851 \\
\hline In-hospital mortality & $16(17.8)$ & $5(12.5)$ & 0.794 \\
\hline Length of inpatient stay & $38 \pm 24$ & $44 \pm 57$ & 0.848 \\
\hline Patients requiring multiple ventriculostomies & $20(22.2)$ & $8(20.0)$ & 0.822 \\
\hline Patients diagnosed with ventriculitis & $14(15.6)$ & $2(5.0)$ & 0.146 \\
\hline Patients requiring shunt insertion & $12(13.3)$ & $9(22.5)$ & 0.205 \\
\hline
\end{tabular}

Values are presented as number (\%) or mean \pm standard deviation.

IV, intravenous; GOS, Glasgow outcome scale. 
that IV aspirin loading within 48 hours of ventriculostomy placement does result in a significantly increased rate of $\mathrm{VAH}$ (30\% incidence in the group treated with IV aspirin). However, the clinical impact of these small foci of radiologically detected hemorrhage needs to be questioned. The maximum hematoma size was $20 \mathrm{~mm}$ and 19 of 27 hemorrhages were below $10 \mathrm{~mm}$. None required surgical treatment. Furthermore, there was no demonstrable difference in mortality or favorable outcome rate between the 2 groups (the control population treated with either no aspirin or low dose oral/ nasogastric aspirin after the coiling procedure). The results highlight a need for careful consideration of the necessity for IV aspirin in this population of predominately poor-grade patients requiring ventriculostomy. Although most bleeds are small and likely have minimal clinical impact, this association could become significant with a larger population group. However, the lack of a clear detrimental clinical effect in this study is reassuring; the results would suggest that where antiplatelet agent use is mandatory (for example when a stent is deployed or there is coil prolapse or platelet aggregation during the procedure) use of IV aspirin will only very rarely result in ventriculostomy-associated bleeding that is of sufficient size to have an impact on outcome or require intervention. Therefore, IV aspirin use should not be withheld out of fear of precipitating $\mathrm{VAH}$ if there is good reason to use it.

In this study, approximately $80 \%$ of patients underwent ventriculostomy placement prior to coiling. Previous studies have assessed the effect of ventriculostomy timing. Some authors have found $\mathrm{VAH}$ rates to be significantly higher when ventriculostomy is performed following aspirin administration ${ }^{9}$ while others have shown a non-significant trend towards increased VAH if ventriculostomy is performed following aspirin administration. 15,16 For those patients drained after administration of antiplatelet agents, platelet infusions are commonly used though this may not alter the rate of associated hemorrhage. ${ }^{17}$

The potential benefits of aspirin at slightly more delayed time points following ventriculostomy and aneurysm securing procedures, particularly during the period most associated with delayed cerebral ischemia $(D C l){ }_{1}^{4}$ likely warrant further investigation. It has been suggested that a potential mechanism of action lies in a reduction in the rate of microthrombosis that may complicate subarachnoid hemorrhage and represent an additional pathophysiological process for $\mathrm{DCl}$ in addition to vasospasm. ${ }^{4}$ In the Magnesium and Aspirin in Subarachnoid Haemorrhage (MASH) study, 100 mg aspirin was administered via a suppository at least 12 hours after the aneurysm securing procedure. In the MASH study, however, a minority of patients were of poor grade (27\% exhibited ventricular hemorrhage), and the majority underwent surgical clipping. The results of a post hoc analysis of the MASH study ${ }^{18}$ imply that patients treated with endovascular coiling had a higher reduction of DCl after aspirin administration compared with patients with neurosurgical clipping. Interestingly, a meta-analysis of the effect of antiplatelet agents on DCl found that as the radiological (Fisher) grade increased the positive impact of anti-platelet agent use diminished. ${ }^{4}$ The majority of the patients in the present investigation were of poor grade with large volumes of cisternal and ventricular blood. Therefore, for this grade of patient, the necessity to treat DCI with aspirin may be less, which may justify delaying or avoiding aspirin use after ventriculostomy insertion.

Our study is limited by its retrospective nature and lack of randomization between study groups. The results reflect our experience at a single neuroscience center and are not universally generalizable to other centers. A limitation of this study is that we cannot fully separate the effects of IV aspirin loading from routine post-procedural low-dose aspirin. The timing of CT head following ventriculostomy insertion was not standardized and usually only performed as clinically indicated so it cannot be stated with certainty whether the recorded VAH occurred before routine low-dose aspirin was commenced. No statistically significant difference in the number of ischemic events was shown between patients loaded with IV aspirin and those who were not; however, the retrospective design of the study precluded accurate assessment of this. Other studies have shown a clear reduction in thrombotic complications with aspirin use. ${ }^{1,2} \mathrm{~A}$ significant confounding factor in this study is that different operators favored more routine IV aspirin administration when compared to others, so the proportion of patients in each group was likely skewed by operator preference rather than a defined indication for IV aspirin administration. Additionally, a platelet-level response to aspirin was not tested.

\section{CONCLUSION}

The findings of this study suggest administration of loading dose of IV aspirin during endovascular treatment of ruptured intracranial aneurysms does significantly increase the risk of VAH, but also that these hemorrhages are small and have 
minimal impact on clinical outcome at discharge. Risk of VAH should not preclude procedural IV aspirin administration if required for an endovascular procedure, but indiscriminate use of IV aspirin should probably be avoided.

\section{Fund}

None.

\section{Ethics Statement}

This study was approved by the Institutional Review Board (IRAS 256424).

The authors mentioned that informed consent for publication was not required because there is no personal identifying information

\section{Conflicts of Interest}

AM undertaken consultancy work for Medtronic and Cerenovus. No other authors have any conflict of interest to disclose.

\section{Author Contributions}

Concept and design: AM and JW. Analysis and interpretation: $D E, R F$, and AM. Data collection: DE, RF, OD, and AM. Writing the article: $D E, R F$, and $A M$. Critical revision of the article: $A M$ and JW. Final approval of the article: AM and JW. Statistical analysis: AM. Overall responsibility: AM.

\section{ORCID}

David Evans: https://orcid.org/0000-0002-7028-9108 Richard Flood: https://orcid.org/0000-0003-0053-2325 Owain Davies: https://orcid.org/0000-0002-4728-6603 James Wareham: https://orcid.org/0000-0002-9009-2230 Alex Mortimer: https://orcid.org/0000-0001-7744-2833

\section{REFERENCES}

1. Edwards NJ, Jones WH, Sanzgiri A, Corona J, Dannenbaum M, Chen PR. Antiplatelet therapy for the prevention of peri-coiling thromboembolism in high-risk patients with ruptured intracranial aneurysms. J Neurosurg 2017;127:1326-1332

2. Ries T, Buhk JH, Kucinski T, Goebell E, Grzyska U, Zeumer H, et al. Intravenous administration of acetylsalicylic acid during endovascular treatment of cerebral aneurysms reduces the rate of thromboembolic events. Stroke 2006;37:1816-1821

3. van den Bergh WM; MASH Study Group, Algra A, Dorhout Mees SM, van Kooten F, Dirven CM, van Gijn J, et al. Randomized con- trolled trial of acetylsalicylic acid in aneurysmal subarachnoid hemorrhage: the MASH study. Stroke 2006;37:2326-2330

4. Cagnazzo F, Derraz I, Lefevre PH, Gascou G, Dargazanli C,

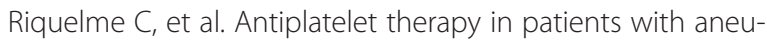
rysmal SAH: impact on delayed cerebral ischemia and clinical outcome. A meta-analysis. AJNR Am J Neuroradiol 2019;40:12011206

5. Ditz C, Machner B, Schacht H, Neumann A, Schramm P, Tronnier VM, et al. Effects of post-interventional antiplatelet therapy on angiographic vasospasm, delayed cerebral ischemia, and clinical outcome after aneurysmal subarachnoid hemorrhage: a single-center experience. [published online ahead of print Jan 25, 2021] Neurosurg Rev 2021

6. Parkhutik V, Lago A, Tembl Jl, Rubio C, Fuset MP, Vallés J, et al. Influence of COX-inhibiting analgesics on the platelet function of patients with subarachnoid hemorrhage. J Stroke Cerebrovasc Dis 2012;21:755-759

7. Meves SH, Neubauer H, Overbeck U, Endres HG. Is there an ideal way to initiate antiplatelet therapy with aspirin? A crossover study on healthy volunteers evaluating different dosing schemes with whole blood aggregometry. BMC Res Notes 2011;4:106

8. Bechan RS, Sprengers ME, Majoie CB, Peluso JP, Sluzewski M, van Rooij WJ. Stent-assisted coil embolization of intracranial aneurysms: complications in acutely ruptured versus unruptured aneurysms. AJNR Am J Neuroradio/ 2016;37:502-507

9. Cagnazzo F, Di Carlo DT, Petrella G, Perrini P. Ventriculostomy-related hemorrhage in patients on antiplatelet therapy for endovascular treatment of acutely ruptured intracranial aneurysms. A meta-analysis. Neurosurg Rev 2020;43:397-406

10. Gardner PA, Engh J, Atteberry D, Moossy JJ. Hemorrhage rates after external ventricular drain placement. J Neurosurg 2009;110:1021-1025

11. Maniker AH, Vaynman AY, Karimi RJ, Sabit AO, Holland B. Hemorrhagic complications of external ventricular drainage. Neurosurgery 2006;59(4 Suppl 2):ONS419-424; discussion ONS424425

12. Sussman ES, Kellner CP, Nelson E, McDowell MM, Bruce SS, Bruce RA, et al. Hemorrhagic complications of ventriculostomy: incidence and predictors in patients with intracerebral hemorrhage. J Neurosurg 2014;120:931-936

13. Müller A, Mould WA, Freeman WD, McBee N, Lane K, Dlugash R, et al.; CLEAR investigators. The incidence of catheter tract hemorrhage and catheter placement accuracy in the clear III trial. Neurocrit Care 2018;29:23-32

14. Hudson JS, Prout BS, Nagahama Y, Nakagawa D, Guerrero WR, 
Zanaty $M$, et al. External ventricular drain and hemorrhage in aneurysmal subarachnoid hemorrhage patients on dual antiplatelet therapy: a retrospective cohort study. Neurosurgery 2019;84:479-484

15. Qin G, Pang G, Zhong S, Chen H, Tang X, Lan S. Increased risk of ventriculostomy-associated hemorrhage in patients treated with antiplatelet agents for stent-assisted coiling of ruptured intracranial aneurysms. Br J Neurosurg 2021;35:270-274

16. Bruder M, Schuss P, Konczalla J, El-Fiki A, Lescher S, Vatter $H$, et al. Ventriculostomy-related hemorrhage after treatment of acutely ruptured aneurysms: the influence of anticoagulation and antiplatelet treatment. World Neurosurg 2015;84:1653-1659

17. Majmundar N, Sarris C, Shastri D, Doran J, Gandhi C, Assina R. Hemorrhagic complications of external ventriculostomy in the aspirin and P2Y12 response assay era. World Neurosurg 2019;122:e961-e968

18. van den Bergh WM, Algra A, Rinkel GJ; MASH Study Group. Magnesium and aspirin treatment in patients with subarachnoid haemorrhage. Comparison of effects after endovascular and neurosurgical aneurysm occlusion. J Neurol 2009;256:213216 